\title{
TAMING OF THE WILD GROUP OF MAGNETIC TRANSLATIONS
}

\author{
PETER VARGA
}

\begin{abstract}
We use a theorem of Auslander and Kostant on the representation theory of solvable Lie-groups for the study of some groups necessary for the description of certain quasi-periodic systems of solid-state physics. We show that the magnetic translation group is tame (Type I) if the magnetic field is not constant but fluctuating.
\end{abstract}

\section{INTRODUCTION}

Lie-groups are divided into two classes (Types I and II) according to the behaviour of their representations [1]. The unitary representations of Type I (tame) groups have essentially unique decompositions into irreducible representations, while in the case of Type II (wild) groups such decomposition can be highly nonuniqe. Finite groups, semisimple and nilpotent Lie-groups are tame, while infinite discrete groups (except those which contain an Abelian subgroup of finite index) are wild. The type of a solvable Lie-group is determined by the behaviour of its coadjoint orbits. According to a theorem of Auslander and Kostant [2], a solvable Lie-group is tame if and only if the set of its coadjoint orbits are separable and the their standard symplectic two-forms are exact. This theorem provides a fairly convenient method to prove the wildness of some solvable groups. The notation of Type I and II representations comes from the theory of von Neumann algebras. This operator algebraic aspect might be especially relevant in physical applications, where one is interested in the properties of the representations of the enveloping algebra. However, we have little to say about this topics in the present paper.

In Kirillov's book [3] two simple examples of wild solvable groups are given. These examples are not just mathematical curiosities, but they emerge naturally in the description of some quasi-periodic systems in solid-state physics. Kirillov's first example has the following physical realization: The functions $a \cos x, a \sin x$, $b \cos \alpha x, b \sin \alpha x$, and the derivation $\partial_{x}$ form a five dimensional Lie-algebra. If $\alpha$ is irrational, then its Lie-group is wild. These operators are the building blocks of the Hamiltonian of an electron moving a quasi-periodic cosine potential.

The Lie-algebra of the second example can be represented by operators which are necessary for the description of the motion of an electron in two dimension under the influence of periodic cosine potentials and uniform magnetic field. The corresponding group contains the magnetic translation group [4, 河].

Date: November 27, 1996.

Key words and phrases. Solvable groups, Type II representations, quasi-periodic systems, solid-state physics . 
The physics of quasi-periodic systems has many characteristic features like the unusual band structure, various types of (de)localisations, etc. [6]. The wildness of the groups in these examples foreshadows the appearance of such features, so the theorem of Auslander and Kostant can be used to predict the qualitative nature of physical systems connected with solvable Lie-groups. In particular, we show that if the Lie-algebra of the magnetic translation group is extended by generators generating fluctuations of the magnetic field, then the corresponding Lie-group becomes tame, so in that case the unusual fractal band structure is not expected.

In the next section we re-present the examples of [3] and give physical realizations of the wild solvable groups. We also determine how the characters of the systems changes if some parameters like the magnitude of the potential and magnetic field is allowed to fluctuate. This paper is basically an extra exercise for the last section of [3].

\section{Solvable Lie-groups in SOlid-State Physics.}

Let us first recall the notation of coadjoint orbits. Let $G$ be a Lie-group, $\mathfrak{g}$ its Lie-algebra, and $\mathfrak{g}^{*}$ its dual. The coadjoint action of $G$ on $\mathfrak{g}^{*}$ is defined by

$$
\left\langle A d_{g}^{*} \xi, A d_{g} X\right\rangle=\langle\xi, X\rangle, \quad \xi \in \mathfrak{g}^{*}, X \in \mathfrak{g}, g \in G
$$

By differentiating 2.1) we obtain

$$
\left\langle a d_{X}^{*} \xi, Y\right\rangle=-\langle\xi,[X, Y]\rangle .
$$

On the orbits $\Omega_{\xi_{0}}=\left\{A d_{g}^{*} \xi_{0}, g \in G\right\} a d_{X}^{*}$ is represented by a vector field $f_{\Omega_{\xi_{0}}}(X)$ - The symplectic two-form $B_{\Omega}$ on $\Omega$ is given by

$$
B_{\Omega_{\xi}}\left(f_{\Omega_{\xi}}(X), f_{\Omega_{\xi}}(Y)\right)(\xi)=\langle\xi,[X, Y]\rangle
$$

A theorem of Auslander and Kostant characterizes the simply connected solvable Type I Lie-groups:

Theorem 1. Let $G$ be a simply connected solvable Lie-group. Then $G$ is Type I (tame) if and only if

1. all coadjoint orbits of $G$ are $G_{\delta}$ sets (i.e. they are countable intersections of open sets) in the usual topology on $\mathfrak{g}$.

2. The symplectic forms $B_{\Omega_{\xi}}$ are exact for all $\xi \in \mathfrak{g}^{*}$.

We use this theorem for the study of some Lie-groups connected with the theory of quasi-periodic systems in solid-state physics.

The simplest example of wild groups is the five dimensional Mautner group [3] consisting of certain $3 \times 3$ complex matrices:

$$
g(t, w, z)=\left(\begin{array}{ccc}
e^{i t} & 0 & z \\
0 & e^{i \alpha t} & w \\
0 & 0 & 1
\end{array}\right), \quad t \in \mathbb{R}, z, w \in \mathbb{C}
$$

where $\alpha$ is a fixed irrational number. The non-zero commutators of the Lie-algebra of this group are

$$
\begin{array}{ll}
{\left[P, S_{1}\right]=C_{1},} & {\left[P, S_{\alpha}\right]=\alpha C_{\alpha}} \\
{\left[P, C_{1}\right]=-S_{1},} & {\left[P, C_{\alpha}\right]=-\alpha S_{\alpha} .}
\end{array}
$$


Operators satisfying the same algebra occur in the theory of one-dimensional quasi-periodic systems. A representation of (2.5) is provided by the following operators acting on $L^{2}(\mathbb{R}, d t)$ :

$$
\begin{array}{lll}
P=\partial_{t}, & S_{1}=a \sin \left(t+\phi_{1}\right), & S_{\alpha}=a_{\alpha} \sin \left(\alpha t+\phi_{\alpha}\right), \\
C_{1}=a \cos \left(t+\phi_{1}\right), & C_{\alpha}=a_{\alpha} \cos \left(\alpha t+\phi_{\alpha}\right) .
\end{array}
$$

A representation with different $a_{1}^{\prime}, a_{\alpha}^{\prime}, \phi_{1}^{\prime}, \phi_{\alpha}^{\prime}$ parameters is isomorphic to (2.6) iff $a_{1}=a_{1}^{\prime}, a_{\alpha}=a_{\alpha}^{\prime}$ and $\phi_{1}-\phi / \alpha=\phi_{1}^{\prime}-\phi^{\prime} / \alpha+2 m \pi+2 n \pi / \alpha$ for some $m, n \in \mathbb{Z}$. One can build the Hamiltonian of an electron moving in a quasi-periodic cosine potential out of these operators:

$$
H=-\frac{1}{2} \partial_{x}^{2}+a_{1} \cos \left(t+\phi_{1}\right)+a_{\alpha} \cos \left(\alpha t+\phi_{\alpha}\right)=-\frac{1}{2} P^{2}+a_{1} C_{1}+a_{\alpha} C_{\alpha} .
$$

In 3] two inequivalent decompositions of the regular representation of (2.4) into irreducible ones are presented. Inequivalent decompositions of a representation of (2.5) occurred in the physics literature, too. It was noted in [7, 8, 9, 10 that although (2.7) has no translational symmetry, it is not completely random either. By adding an extra dimension, translations by $2 \pi$ and $2 \pi / \alpha$ can be executed in separate dimensions. For that purpose, we consider the following representation of (2.5) on $L^{2}\left(\mathbb{R}^{2}, d x d y\right)$ :

$$
\begin{array}{lll}
P=\partial_{x}+\partial_{y} & S_{1}=a_{1} \sin x, & S_{\alpha}=a_{\alpha} \sin \alpha y, \\
& C_{1}=a_{1} \cos x, & C_{\alpha}=a_{\alpha} \cos \alpha y .
\end{array}
$$

Since $P$ is the generator of translations only along the lines $l_{c}: y=x+c$, the representation (2.10) is decomposable into irreducible representations acting on the Hilbert-spaces $L^{2}\left(l_{c}\right)$. These representations are isomorphic to (2.6) with parameters $\phi_{1}=0, \phi_{\alpha}=c \alpha$. A different decomposition of $L^{2}\left(\mathbb{R}^{2}, d x d y\right)$ is based on the periodicity of (2.8) on the $x y$-plane. The operator $H=-1 / 2 P^{2}+a_{1} C_{1}+a_{\alpha} C_{\alpha}$ is indeed invariant against the translations $(x, y) \rightarrow(x+2 \pi, y)$ and $(x, y) \rightarrow$ $(x, y+2 \pi / \alpha)$. The translational symmetry entails the existence of Bloch wavefunctions

$$
\psi(x+2 \pi, y)=e^{i s} \psi(x, y), \quad \psi\left(x, y+\frac{2 \pi}{\alpha}\right)=e^{i t} \psi(x, y) .
$$

The operators acting on such wave-functions for fixed $s$ and $t$ provide exactly the infinitesimal form of the irreducible representation occurring in the second decomposition of the regular representation in [3]. Indeed, if we introduce the periodic functions

$$
\tilde{\psi}(x, y)=e^{-i(s x+t \alpha y)} \psi(x, y),
$$

then the operators (2.8) act on $\tilde{\psi}$ as

$$
\tilde{P}=\partial_{x}+\partial_{y}+i(s+\alpha t), \quad\left(S_{1}, C_{1}, S_{\alpha}, C_{\alpha} \text { are unchanged }\right) .
$$

Since $\tilde{\psi}$ is periodic, we can regard it as a function defined on the torus $S^{1} \times S^{1}=[0,2 \pi) \times[0,2 \pi / \alpha)$. The action of the operators 2.11$)$ on $L^{2}\left(S^{1} \times S^{1}\right)$ is irreducible. The existence of a representations with Bloch wave-functionals does not a priori implies the occurance of extended states in the physical representation (2.8). Indeed, as it was stressed in [11], inequivalent representations of the same algebra might have very different spectral and localizational properties. Nevertheless, the existence of extended states in this system was established in [12, 13, 14. 
Next we study the effect of the fluctuation of the magnitude of the potential. For this purpose we add the generator $M=\partial_{a}$ to the operators of (2.6). $M$ changes the amplitudes of the potentials $S_{1}$ and $C_{1}$. To keep the algebra closed we need to add the operators $S_{0}=\sin \left(t+\phi_{1}\right)$ and $C_{0}=\cos \left(t+\phi_{1}\right)$ to $(2.6)$, too. The extra non-zero commutators (compared to (2.5)) of the extended Lie-algebra $\mathfrak{g}$ are

$$
\left[P, S_{0}\right]=C_{0}, \quad\left[P, C_{0}\right]=-S_{0}, \quad\left[M, S_{1}\right]=S_{0}, \quad\left[M, C_{1}\right]=C_{0} .
$$

The Lie-group $G$ of $\mathfrak{g}$ has a representation by $4 \times 4$ matrices

$$
g(t, a, u, w, z)=\left(\begin{array}{cccc}
e^{i t} & a & 0 & u \\
0 & e^{i t} & 0 & z \\
0 & 0 & e^{i \alpha t} & w \\
0 & 0 & 0 & 1
\end{array}\right), \quad a, t \in \mathbb{R}, u, w, z \in \mathbb{C}
$$

If $\mathfrak{g}^{*}$ is represented by matrices of the following form

$$
\xi(\tau, p, l, m,)=\left(\begin{array}{cccc}
i \tau & 0 & 0 & 0 \\
p & 0 & 0 & 0 \\
0 & 0 & 0 & 0 \\
l & m & n & 0
\end{array}\right), \quad \tau, t \in \mathbb{R}, l, m, n \in \mathbb{C},
$$

so the pairing between $\mathfrak{g}$ and $\mathfrak{g}^{*}$ is

$$
\langle\xi, h\rangle=\Re(\operatorname{Tr}(\xi h)), \quad \xi \in \mathfrak{g}^{*}, h \in \mathfrak{g},
$$

then the coadjoint action is

$$
\begin{aligned}
& A d_{g(t, a, u, w, z)}^{*} \xi(\tau, p, l, m, n)= \\
& \quad \xi\left(\tau+\Im(l u+z m+\alpha n w), p-\Re(l z), l e^{-i t}, m e^{-i t}-\Re(l z), n e^{-i \alpha t}\right) .
\end{aligned}
$$

The four dimensional orbits are given by the parametric equations

$$
l=l_{0} e^{i t}, \quad n=n_{0} e^{i \alpha t} .
$$

Since the orbits are dense subsets of the sets

$$
|l|=l_{0}, \quad|n|=n_{0}
$$

the first criteria of the Auslander-Kostant theorem fails, so the group remains wild despite the fluctuation of the potential.

In the following we turn our attention to Kirillov's second example of wild groups. This group is closely related to the magnetic translation group, whose Type II nature at irrational magnetic flux was pointed out by [15] This is a seven dimensional Lie-algebra whose nonzero commutators are

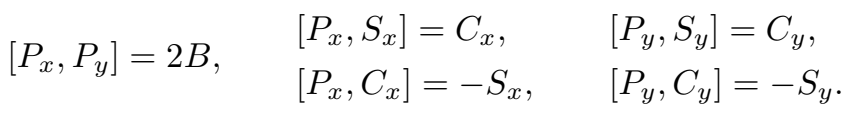

This algebra is represented by the operators

$$
\begin{array}{lll}
\hat{P}_{x}=i \partial_{x}-b y, & \hat{C}_{x}=\cos x, & \hat{C}_{y}=\cos y, \\
\hat{P}_{y}=i \partial_{y}+b x, & \hat{S_{x}}=\sin x, & \hat{S_{y}}=\sin y,
\end{array}
$$

on $L^{2}\left(\mathbb{R}^{3}, d x d y d z\right)$. The Hamiltonian of an electron moving in constant magnetic field in a periodic crystal can be formed out of these operators:

$$
\hat{H}=\hat{P}_{x}^{2}+\hat{P}_{y}^{2}+\hat{C}_{x}+\hat{C}_{y}
$$


If we regard the generators as linear functions on $\mathfrak{g}^{*}$, then the coadjoint orbits are

$$
C_{x}^{2}+S_{x}^{2}=r_{1}^{2}, \quad C_{y}^{2}+S_{y}^{2}=r_{2}^{2}, \quad B=r_{3} .
$$

If the orbits are parametrized as

$$
C_{x}=r_{1} \cos \phi, \quad S_{x}=r_{1} \sin \phi, \quad C_{y}=r_{2} \cos \psi, \quad S_{y}=r_{2} \sin \psi,
$$

then the symplectic two-form $B_{\Omega}$ is

$$
B_{\Omega}=d \phi \wedge d P_{x}+d \psi \wedge d P_{y}+2 r_{3} d \phi \wedge d \psi
$$

Since

$$
\int_{\left\{P_{x}=P_{y}=0, B=r_{3}\right\}} B_{\Omega}=8 \pi^{2} r_{3},
$$

$B_{\Omega}$ is not exact, so the second criteria of the Auslander-Kostant theorem fails, consequently the group of magnetic translations is wild.

Now let us see what happens if the external magnetic field is dynamical, too. To describe the fluctuation of $b$ we extend the set of generators $(2.22)$ by $\hat{E}=i \partial_{b}$. In order to keep the commutators closed, we need to adjoin the operators $\hat{Y}=$ $-i\left[\hat{E}, \hat{P}_{x}\right]$ and $\hat{X}=i\left[\hat{E}, \hat{P}_{y}\right]$, too. So the following eleven-dimensional Lie-algebra is necessary to describe the coupled system of an electron and the fluctuating external magnetic field:

$$
\begin{gathered}
{\left[P_{x}, S_{x}\right]=C_{x}, \quad\left[P_{y}, S_{y}\right]=C_{y}, \quad\left[P_{x}, X\right]=I,} \\
{\left[P_{x}, C_{x}\right]=-S_{x}, \quad\left[P_{y}, C_{y}\right]=-S, \quad\left[P_{y}, Y\right]=I,} \\
{\left[E, P_{x}\right]=-Y, \quad\left[P_{x}, P_{y}\right]=2 B,} \\
{\left[E, P_{y}\right]=X, \quad[E, B]=I .}
\end{gathered}
$$

If we use the generators of the Lie-algebra as linear functions on $\mathfrak{g}^{*}$ then the coadjoint action corresponds to the following vector fields:

$$
\begin{array}{ll}
V_{P_{x}}=-C_{x} \partial_{S_{x}}+S_{x} \partial_{C_{x}}+2 B \partial_{P_{y}}+Y \partial_{E}+I \partial_{Y}, \\
V_{P_{y}}=-C_{y} \partial_{S_{y}}+S_{y} \partial_{C_{y}}-2 B \partial_{P_{x}}-X \partial_{E}+I \partial_{X}, \\
V_{S_{x}}=C_{x} \partial_{P_{x}}, & V_{S_{y}}=C_{y} \partial_{P_{y}}, \\
V_{C_{x}}=-S_{x} \partial_{P_{x}}, & V_{C_{y}}=-S_{y} \partial_{P_{y}}, \\
V_{B}=-I \partial_{E}, & V_{E}=I \partial_{B}-Y \partial_{P_{x}}+X \partial_{P_{y}}, \\
V_{X}=-I \partial_{P_{x}}, & V_{Y}=-I \partial_{P_{y}},
\end{array}
$$

Note that $\partial_{I}$ does not occur in these expressions, so $I=I_{0}=$ const. on each orbit. The form of $V_{P_{x}}$ and $V_{P_{y}}$ implies that

$$
C_{x}^{2}+S_{x}^{2}=r_{x}^{2}, \quad C_{y}^{2}+S_{y}^{2}=r_{y}^{2},
$$

while 2.28 ) entails

$$
\mathcal{L}\left(\left\{V_{X}, V_{Y}, V_{E}, V_{B}\right\}\right)=\mathcal{L}\left(\left\{\partial_{P_{x}}, \partial_{P_{y}}, \partial_{E}, \partial_{B}\right\}\right) .
$$

So the orbits are generated by the vectors $\partial_{P_{x}}, \partial_{P_{y}}, \partial_{E}, \partial_{B}$ and by

$$
\tilde{V}_{P_{x}}=-C_{x} \partial_{S_{x}}+S_{x} \partial_{C_{x}}+I_{0} \partial_{Y}, \quad \tilde{V}_{P_{y}}=-C_{y} \partial_{S_{y}}+S_{y} \partial_{C_{y}}+I_{0} \partial_{x} .
$$


The integral manifolds of these vectors are

$$
\begin{array}{lll}
C_{x}=r_{x} \cos \phi, & S_{x}=r_{x} \sin \phi, & Y=I_{0}\left(\phi+\phi_{0}\right) \\
C_{y}=r_{y} \cos \psi, & S_{y}=r_{y} \sin \psi, & X=I_{0}\left(\psi+\psi_{0}\right),
\end{array}
$$

while $E, B, P_{x}, P_{y}$ are arbitrary. So the maximal dimensional orbits are homeomorph to $\mathbb{R}^{6}$. Since $H^{2}\left(\mathbb{R}^{6}\right)=0$, the symplectic two-form $B_{\Omega}$ is necessarily exact. Consequently this extension of the magnetic translation group is tame!

Acknowledgements: We are grateful to Prof. István Kovács for discussions on operator algebras. We thank the referee for helpful suggestions and for calling our attention to some references. This research was partially funded by the grant OTKA-F015470.

\section{REFERENCES}

[1] G.W.Mackey, Unitary Groups Representations, Benjamin, Reading, 1978.

[2] L.Auslander,B.Kostant, Bull.Amer.Math.Soc. 73 (1967) 692.

[3] A.A.Kirillov, Lectures on the Theory of Group Representations, Springer-Verlag, BerlinHeidelberg-New York, 1976.

[4] J.Zak, Phys.Rev. A134 (1964) 1602.

[5] D.R.Hofstadter, Phys.Rev. B14 (1976) 2239.

[6] J.B.Sokoloff, Phys.Rep. 126 (4), (1985) 189-244.

[7] M.V.Romerio, J.Math.Phys. 12 (1971) 552.

[8] P.M.deWollf, Acta Cryst. A30 (1974) 777.

[9] P.M.deWollf,T.Janssen and A.Janner, Acta Cryst. A37 (1981) 625.

[10] A.Janner,T.Jannsen, Phys.Rev. B15 (1977) 643.

[11] J.Bellissard, in ed.S.Graffi, Transition to Chaos in Classical and Quantum Mechanics, Lecture Notes in Mathematics 1589, Spinger-Verlag, Berlin-Heidelberg-New York, 1991.

[12] J. Bellissard, R. Lima, D. Testard, Commun. Math. Phys. 88, 207-234 (1983).

[13] Ya. G. Sinai, J. Stat. Phys. 46, 861-909 (1987).

[14] J. Fröhlich, T. Spencer, P. Wittwer, Commun. Math. Phys. 132, 5-25 (1990).

[15] A. Grossman, in 1971 Europhysics Conference on Statistical Mechanics and Field Theory, Haifa, R.N. Sen \& C. Weil Eds (1972). See also F.Bentosela, Lett. Nuovo Cim. B 16, 115-126 (1973).

Institute of Mathematics and Informatics, Lajos Kossuth University, H-4010 DebreCEN, Hungary

E-mail address: varga@@math.klte.hu 